\section{BSF-2 is not just a differentiation factor}

SIR-An interesting point has escaped Hirano et al. ' in their report of the sequence of BSF-2, a B-cell differentiation factor. The point is that the sequence of BSF2 is identical to that recently described by Haegeman et al. ${ }^{2}$ of the State University of Ghent for a molecule called $26 \mathrm{~K}$. This molecule was originally described as a byproduct of interferon- $\beta_{1}$ (IFN- $\beta_{1}$ ) produced in human fibroblasts ${ }^{3.4}$, and called IFN- $\beta$, after investigators at the Weizmann Institute detected antiviral activity associated with the molecule . $^{4}$

In both laboratories the primary structure of the $26 \mathrm{~K} / \mathrm{IFN}-\beta_{2}$ molecule has been determined by molecular cloning of the complementary $\mathrm{DNA}^{2.5}$. But the question of whether the natural $26 \mathrm{~K} / \mathrm{IFN}-\beta$, product possesses antiviral activity has remained a matter of controversy ${ }^{3}$. Hence, its true biological function has remained in question

A first hint at an answer to this question came from the observation both in the laboratory of $\mathrm{J}$. Content at the Pasteur Institute in Brussels and in our own laboratory that the mRNA for the $26 \mathrm{~K} / \mathrm{IFN}-\beta_{2}$ molecule is induced in fibroblasts by interleukin-1 (IL-1) ${ }^{6}$. This suggested that the molecule might be one of the mediators of the multiple biological effects of IL-1. In an extensive search for biologically active substances released from fibroblasts by treatment with IL-1, Van Damme from our laboratory, together with J. Van Snick of the Ludwig Institute in Brussels discovered that fibroblasts exposed to treatments that induce appearance of the mRNA for $26 \mathrm{~K} / \mathrm{IFN}$ $\beta_{2}$, release a potent growth factor for Bcell hybridomas and plasmacytomas ${ }^{7.8}$. In an effort to demonstrate whether this hybridoma/plasmacytoma growth factor (HPGF) is identical to the $26 \mathrm{~K} / \mathrm{IFN}-\beta_{2}$ molecule, these investigators found that the mRNA for HPGF hybridizes to a cDNA probe of $26 \mathrm{~K} / \mathrm{IFN}-\beta_{2}{ }^{8}$. Subsequently, HPGF was purified to homogeneity and, in collaboration with R. Simpson of the Ludwig Institute in Melbourne, its amino terminus was sequenced. This sequence matches exactly that predicted from the $26 \mathrm{~K}$ cDNA clones and allows us to identify exactly the cleavage point between the signal peptide and the mature protein.

Recently there has been renewed interest in the possibility that the molecule may have direct antiviral activity ${ }^{4}$. Two cytokines, IL-1 and tumour necrosis factor $(\mathrm{TNF}-\alpha)$, which are both able to induce the mRNA for the factor ${ }^{6,9}$, also possess antiviral activity that can be neutralized by antisera against interferon- $\beta$. The question is whether these antiviral effects are mediated by IFN- $\beta_{1}$ or IFN- $\beta$.
By using the B-cell promoting effect as an assay system, we have succeeded in preparing an antiserum that reacts with $26 \mathrm{~K} /$ IFN- $\beta$, but not with IFN- $\beta_{1}$. This antiserum fails to neutralize the antiviral effect of IL-1 and TNF (J. Van Damme et al., unpublished data). In contrast, sera that are specific for IFN- $\beta$, and do not neutralize the HPGF activity of $26 \mathrm{~K} / \mathrm{IFN}$ $\beta_{2}$, do neutralize the antiviral effects of IL-1 and TNF ${ }^{16}$. This clearly demonstrates that IFN $-\beta_{2}$ does not play a role in the antiviral effect of IL-1 or TNF.

In conclusion, the substances known as BSF-2, IFN- $\beta_{2} 26 \mathrm{~K}$ and HPGF are one and the same. The substance certainly qualifies as a B-cell differentiation and growth factor but has little, if any, antiviral activity.

\section{The Rega Institute,}

University of Leuven,

B-3000 Leuven, Belgium

1. Hirano, T. et al. Nature 324, $73-76$ (1986).

2. Haegeman, G. et al. Eur. J. Biochem. 159, 625-632 (1986). . Content, J, et al. Proc nain. Acad. Sci. U.S.A. 79, 27682772 (1982)

4. Weissenbach, J et al. Proc natn. Acad. Sci. U.S.A. 77 $7152-7156(1980)$.

5. Revel, M. et al. in The Biology of the Interferon System (eds Stewart, W.E. \& Schellekens, H.) 207-216 (Elsevier, Amsterdam, 1986).

6. Content, J. et al. Eur. J. Biochem. 152, 253-257 (1985)

7. Van Damme, J. et al. Eur. J. Immun. (in the press)

8. Van Damme, J. et al. J. exp. Med. (submitted)

. Kohase M et al Cell 45, 659-666 (1986)

10. Van Damme, J. et al. Nature 314, 266-268 (1985)

\section{Plant tumour induction}

SIR-In Conrad Lichtenstein's otherwise excellent short comment on tumour induction by Agrobacterium tumefaciens', he omitted to mention the biological context of the bacterium. Wounding is a prerequisite for tumour formation, the basis for which is that wounded cells release inducers, such as acetosyringone, that activate the vir-region of the Ti plasmid of the bacterium, and thus facilitate its $\mathrm{T}$ DNA transfer. A. tumefaciens occurs in the rhizosphere, and has been demonstrated to bind to plant cells. This implies that the bacterium is attracted to plants, but the biochemical basis for this has been left unexplained.

Intrigued, we set out to investigate chemotaxis in Agrobacterium. Using the methods of Adler ${ }^{2.3}$, we have demonstrated that $A$. tumefaciens is capable of chemotaxis towards a range of phenolic plant wound exudates ${ }^{4}$ including vanillyl alcohol. Crucially, we have discovered that chemotaxis towards the acetosyringone is determined by the Ti-plasmid. Interestingly, the chemotactic optimum occurs at $100 \mathrm{nM}$ acetosyringone, some 100 -fold lower than the concentration giving maximal vir-induction.

This suggests a biological system in which acetosyringone, at low concentrations, acts as a chemoattractant for $A$. tumefaciens. The bacterium thus moves up the concentration gradient towards the wounded plant cells. Then at higher concentrations, acetosyringone causes vir-induction, and ultimately T-DNA transfer.

Charles H. Shaw

Alison M. Ashby MARTIN D. WATSON

Department of Botany,

University of Durham,

South Road, Durham, DHI 3LE, UK

Lichtenstein. C. Nature 322, 682-683 (1986)

2. Adler. J. Science 166, 1588-1597 (1969)

3. Adler, J. J. gen. Microbiol. 74, 77-91 (1973)

4. Ashby, A.M., Watson. M.D. \& Shaw. C.H. FEMS Micro. Letts (in the press)

Stachel, S.E., Messens, E., Van Montagu. M. \& Zambryski, P. Nature 318, 624-629 (1985).

\section{An alternative view of the origin of life}

Sir-In his News and Views item on the discovery of enzymatic activities in RNA molecules, Gilbert goes on to "contemplate an RNA world, containing only RNA molecules that serve to catalyse the synthesis of themselves"1. But what would be the source of energy in this RNA-run world? Another, and more basic question is, all these RNAs to what end? Are we interested in finding mere self-replicating machinery as an answer to the origin of life, or are we looking for a prototype of the self-propagating form as it exists in an organism?

At the simplest level, life is a massive molecular network that is able to gather a few substances to make usable energy and more molecules of different types that permit the replication of genetic material, which contains the information to run the network. The genetic material, the encoded proteins and the intermediary RNAs have received ready recognition from those engaged in the study of origin of life and its evolution; the tiny metabolites whose numerous linear, branched and cyclical conversions constitute the network, have received next to no attention. This is like embarking on the study of a kingdom and concentrating only on the king's family.

Enzymes can conceivably arise as the result of the formation of appropriate sequences of nucleic acids by random associations of nucleotides; but what function (and hence what selection value) can any of them have if there is no substrate to act upon? And, where does the substrate come from and what happens to the product? Meaningfulness demands a full pathway; but a pathway by itself is as meaningless as an enzyme in isolation. Pathways have to be able to interact with each other. The hypothesis of the formation of life by random associations of nucleotides and selection suffers from the need for prior and continual (for who knows when the gene encoding the proper 